\title{
GENERALIZATION OF A THEOREM OF OSGOOD TO THE CASE OF CONTINUOUS APPROXIMATION ${ }^{1}$
}

\section{A. M. OSTROWSKI}

In the historical development of the theory of convergence the following theorem of Osgood ${ }^{2}$ has played a very important role: $S u p$ pose that the functions $f_{\nu}(x)$ are continuous in the interval $(a, b)$ and with $\nu \rightarrow \infty$ converge to a limit $f(x)$ which is also continuous in $(a, b)$; then to any $\epsilon>0$ there exists a subinterval $\left(a^{\prime}, b^{\prime}\right)$ of $(a, b)$ and an integer $n_{0}$ such that

$$
\left|f_{\nu}(x)-f(x)\right|<\epsilon
$$

is valid in $\left(a^{\prime}, b^{\prime}\right)$ for all $\nu>n_{0}$.

Although since 1897 a great number of results in this direction have been found, Osgood's theorem has not been as yet completely superseded and provides sometimes a useful means in dealing with convergent sequences. In what follows I prove an analogous theorem for the case of continuous convergence.

THEOREM. Let $f(t, x)$ be a continuous function of the point $(t, x)$ for $a<x<b$ and $t \geqq T$ and suppose that we have

$$
\lim _{t \rightarrow \infty} f(t, x)=f(x)
$$

where $f(x)$ is also continuous in $(a, b)$; then for any $\epsilon>0$ there exists a subinterval $J$ of $(a, b)$ and a number $T_{0}$ such that we have for $x<J$, $t \geqq T_{0}$ :

$$
|f(t, x)-f(x)|<\epsilon \quad\left(t \geqq T_{0}, x<J\right) .
$$

The proof of our theorem follows easily from the following

Lemma. If $f(t, x)$ is a continuous function of the point $(t, x)$ for $t \geqq T$ and $a^{\prime \prime}<x<b^{\prime \prime}$, where $T$ is a positive integer, then a seguence of positive numbers $t_{v}$ tending to $\infty$ can be found such that to each $t \geqq T$ there corresponds a t, tending to $\infty$ with $t$, for which we have

$$
\left|f(t, x)-f\left(t_{v}, x\right)\right| \leqq 1 / t .
$$

Proof of the Lemma. Since $f(t, x)$ is uniformly continuous in any

Received by the editors July 1, 1949.

${ }_{1}$ The preparation of this paper was sponsored by the Office of Naval Research.

2 W. F. Osgood, Non-uniform convergence and the integration of series term by term, Amer. J. Math. vol. 19 (1897) p. 161, the "Fundamental Theorem." 
rectangle $a^{\prime \prime} \leqq x \leqq b^{\prime \prime}, n \leqq t \leqq n+1$, for $n \geqq T$, there corresponds to any integer $n \geqq T$ a positive integer $N_{n}$ such that we have

$$
\left|f(t, x)-f\left(t_{0}, x\right)\right| \leqq \frac{1}{n+1}
$$

if $t$ and $t_{0}$ lie between $n$ and $n+1$ and $\left|t-t_{0}\right| \leqq 1 / N_{n}, a^{\prime \prime} \leqq x \leqq b^{\prime \prime}$. We subdivide all intervals $\langle n, n+1\rangle$ with $n \geqq T$ in $N_{n}$ equal parts and denote the division points from $t_{1}=T$ on by $t_{1}, t_{2}, \ldots$. Then for each $t \geqq T$ there exists a $t_{\nu}$ from the interval between $[t]$ and $[t]+1$ such that $t_{\nu} \leqq t<t_{\nu+1}$ and therefore by (2) for all $x$ in $\left\langle a^{\prime \prime}, b^{\prime \prime}\right\rangle$

$$
\left|f(t, x)-f\left(t_{\nu}, x\right)\right| \leqq \frac{1}{\left[t_{\nu}\right]+1} \leqq \frac{1}{t} .
$$

Our lemma is proved.

Proof of THE THEOREM. Without loss of generality $T$ in the theorem can be supposed a positive integer. Apply the lemma to a closed interval $\left\langle a^{\prime \prime}, b^{\prime \prime}\right\rangle$ contained in $(a, b)$ and form for this closed interval and the function $f(t, x)$ the sequence $t_{\nu}$ of the lemma. In applying Osgood's theorem to the sequence of functions $f\left(t_{v}, x\right)$, to a given $\epsilon>0$ we find a subinterval $J$ of $\left\langle a^{\prime \prime}, b^{\prime \prime}\right\rangle$ and an integer $n_{0}$ such that

$$
\left|f\left(t_{v}, x\right)-f(x)\right| \leqq \epsilon / 2 \quad\left(\nu \geqq n_{0}, x<J\right) .
$$

Take then $T_{0}$ such that $1 / T_{0}<\epsilon / 2$ and such that for each $t \geqq T_{0}$, a $t_{\nu}$ corresponding to $t$ by the lemma has an index $\geqq n_{0}$. Then we have

$$
\left|f(t, x)-f\left(t_{v}, x\right)\right| \leqq 1 / t \leqq 1 / T_{0}<\epsilon / 2
$$

for all $x$ from $\left\langle a^{\prime \prime}, b^{\prime \prime}\right\rangle$ and the assertion (1) follows from the inequalities (3) and (4). Our theorem is proved.

Both the theorem and the lemma proved remain valid if $t$ goes to $\infty$ through a set $M$ which has a closed intersection with any finite interval. The necessary modifications in the proof are obvious. The case of a finite limiting point for $t$ is reduced to the case treated above by a linear transformation.

National Bureau of Standards and UNIVERSITY OF BASLE 